\title{
Step height measurement of surface-functionalized micro-machined micro-cantilever by scanning white light interferometry
}

\author{
A S Kurhekar ${ }^{1,2^{*}}$ and Prakash R Apte ${ }^{1,2}$
}

\begin{abstract}
Micro-cantilever arrays with different dimensions are fabricated by micro-machining technique onto a silicon $<100>$ substrate. These sputtered gold-coated micro-cantilevers were later surface-functionalized. Scanning electron microscopy, atomic force microscopy (AFM), and optical diffraction using a laser source are employed to characterize the morphology and image measurement of the micro-cantilever arrays. The spatial resolution produced in the proposed image measurement method is approaching $1 \mu \mathrm{m}$, and the repeatable precision is nanometer confirmable. Compared with conventional AFM and SPM measurement techniques, the proposed method has demonstrated sufficient flexibility, repeatability, and reliability. The experimental results have been analyzed and presented in this paper for microelectromechanical system (MEMS) micro-cantilevers. The scanning white light interferometry-based two-point high-resolution optical method is presented for characterizing micro-cantilevers and other MEMS micro-structures. In this letter, we investigate the micro-structure fabrication and image measurement of length, width, and step height of micro-cantilever arrays fabricated using bulk micro-machining technique onto a silicon $<100>$ substrate.
\end{abstract}

Keywords: Scanning electron microscopy, Atomic force microscopy, Micro-cantilever, Optics, Image measurement, Silicon $\langle 100\rangle$, Scanning white light interferometry

\section{Background}

Accurate measurement of dimensions of micro-structures has recently received much attention because of their potential advantages over conventional atomic force microscopy (AFM)/SPM techniques [1]. A common method to fabricate micro-cantilevers is to pattern the deposited continuous film using bulk or surface micromachining technique [1,2]. However, these methods are demonstrated perfect only for sub-micron micro-cantilever arrays. As the micro-cantilever size decreases to nanometer, an interesting behavior may be expected. In particular, reduced micro-cantilever size results in changes of the domain structure [2] and will affect the characterization of the micro-cantilever. One of the other methods to fabricate nanometer micro-cantilever arrays is by laser micro-machining the deposited material onto a silicon

\footnotetext{
* Correspondence: askurhekar@gmail.com

'Department of Electrical Engineering, Indian Institute of Technology Bombay, Powai, Mumbai 400076, India

${ }^{2}$ Datta Meghe College of Engineering, Airoli, Navi Mumbai 400708, India
}

substrate [2,3]. Until now, the conventional image measurement technique for planar micro-structural properties of micro-cantilevers on silicon $<100>$ substrates has been studied [4]. However, the applicability of optical methods for micro-structure arrays is widely established. In this letter, we investigate the micro-structure fabrication and image measurement of length, width, and step height of micro-cantilever arrays fabricated using bulk micromachining technique onto a silicon $\langle 100\rangle$ substrate.

Step height measurement is required in many fields including semiconductors, micro-circuitry, and printing. Small steps are often measured using a profilometer and by calculating the least squares straight line through the data and then identifying the areas above and below this as the step and the substrate, respectively. The step height is calculated using least squares fit in the equation $Z=a X+b+h \hat{I}^{\prime}$, where $a, b$, and $h$ are unknown and $\hat{I}^{\prime}$ takes the value of +1 in the higher regions and -1 in the lower regions. The unknown $a$ and $b$ represent the slope and the intercept of the line, respectively. The step height 
Table 1 Dimensions of micro-cantilever: designed and measured using the proposed method

\begin{tabular}{lccc}
\hline Serial number & \multicolumn{3}{c}{ Micro-cantilever } \\
\cline { 2 - 4 } & Dimensions & Designed & $\begin{array}{c}\text { Measured after } \\
\text { micro-machining }\end{array}$ \\
\hline 1 & Length & $200 \mu \mathrm{m}$ & $184 \pm 0.01 \mu \mathrm{m}$ \\
2 & Width & $60 \mu \mathrm{m}$ & $50 \pm 0.01 \mu \mathrm{m}$ \\
3 & Step height & $200 \mathrm{~nm}$ & $180 \pm 0.01 \mathrm{~nm}$ \\
\hline
\end{tabular}

is calculated as twice the value of the third unknown, $h$. This approach is fine for samples where the flatness of the step and the substrate are both good.

\section{Methods}

Two procedures were used to fabricate micro-cantilever arrays. Firstly, silicon $<100>$ substrates were deposited as silicon dioxide using a thermal oxidization procedure as described previously [5]. The oxide deposition rate was about $1.5 \mathrm{~nm} / \mathrm{min}$, and the gas flow rate was $18 \mathrm{sccm}$. After oxidization and patterning, the residual silicon was removed using an anisotropic etchant, TMAH, at $80^{\circ} \mathrm{C}$ for $45 \mathrm{~min}$ [6]. Secondly, micro-cantilever arrays were deposited by radio-frequency (RF) magnetron sputtering from a gold target at room temperature. The sputtering chamber was firstly pumped down to 180 mTorr. Then, the deposition of chrome was carried out under Ar atmosphere with about 180 mTorr and a gas flow rate of $18 \mathrm{sccm}$. During the deposition process, the continuous film of gold [4] was also deposited onto a silicon substrate under the same condition for the convenience of measuring the film thickness.

The sputtered chrome-gold layer is known to exhibit affinity with thiophenol molecules $[7,8]$. Considering this fact, we have dip-coated the piezoresistive microcantilevers with $1 \mu \mathrm{mol}$ thiophenol in ethanol solution for $3 \mathrm{~h}$ and then rinsed with ethanol for $2 \mathrm{~min}$. The surface becomes functionalized.

The surface morphology of the micro-cantilever arrays was investigated by scanning electron microscopy

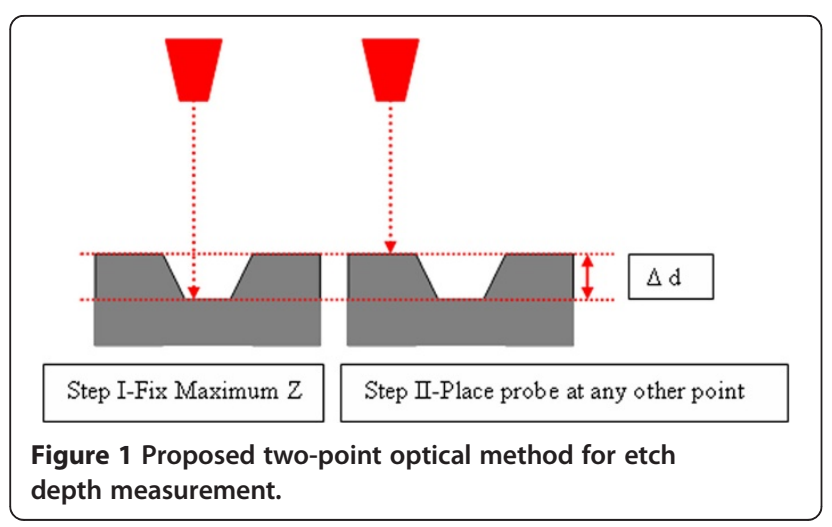

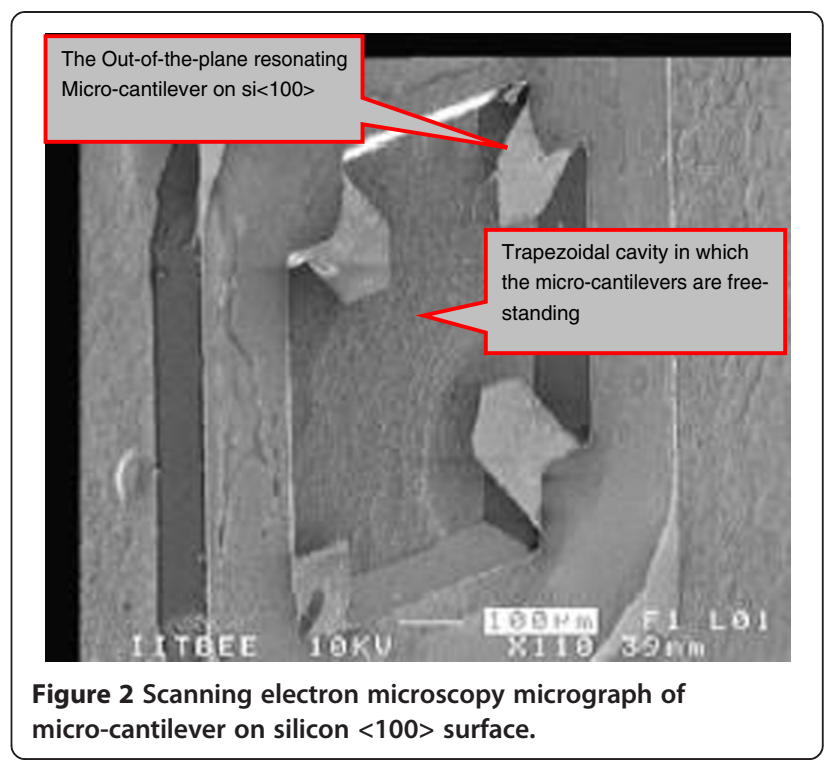

(SEM; JEOL 2000, Akishima-shi, Japan). The grazing incidence laser diffraction, which avoids the effect of the substrate to the pattern, was used to study the image measurement of the micro-structure. The mechanical properties at the temperature $300 \mathrm{~K}$ were measured by atomic force microscopy.

Image measurement of significant parameters of surfacefunctionalized micro-machined micro-cantilevers such as length, width, and step height was obtained using the SEEBREZ ${ }^{\circledast}$ optical multi-sensing system with laser probe (Quality Control Solutions, Inc., Temecula, CA, USA) and Taylor Hobson's Form Talysurf ${ }^{\oplus}$ 3-D surface profiler machine (Leicester, UK) with 3-D Telemap Gold 3-D profile software. The coni-spherical stylus with a base radius of $2 \mu \mathrm{m}$ was used for the contact-mode measurements.

The coordinate measurements were done with the SEEBREZ ${ }^{\odot}$ optical multi-sensing system with a laser probe. This system has an autofocus facility. After the sample was prepared for measurements, the origin of the wafer coordinates was put relatively. Then, the maximum $Z$ coordinate was fixed with a 633-nm laser beam marker, so the maximum step that can be measured is $633 / 4=158.25 \mathrm{~nm}$. The measurement of length and width is relatively simple because the two points shall be placed in-plane. However, for the measurement of step height, the two points shall not be in-plane. So, we fix one point (maximum $Z$ ) using the marker, and the other point can be placed where the height needs to be measured (minimum $Z$ ). The difference between maximum $Z$ and minimum $Z$ of the markers $(\Delta d)$ shall give the step height. The change in the film step height $(\Delta d)$ is directly proportional to the wavelength of the laser light and is inversely proportional to twice the refractive index of the film being 


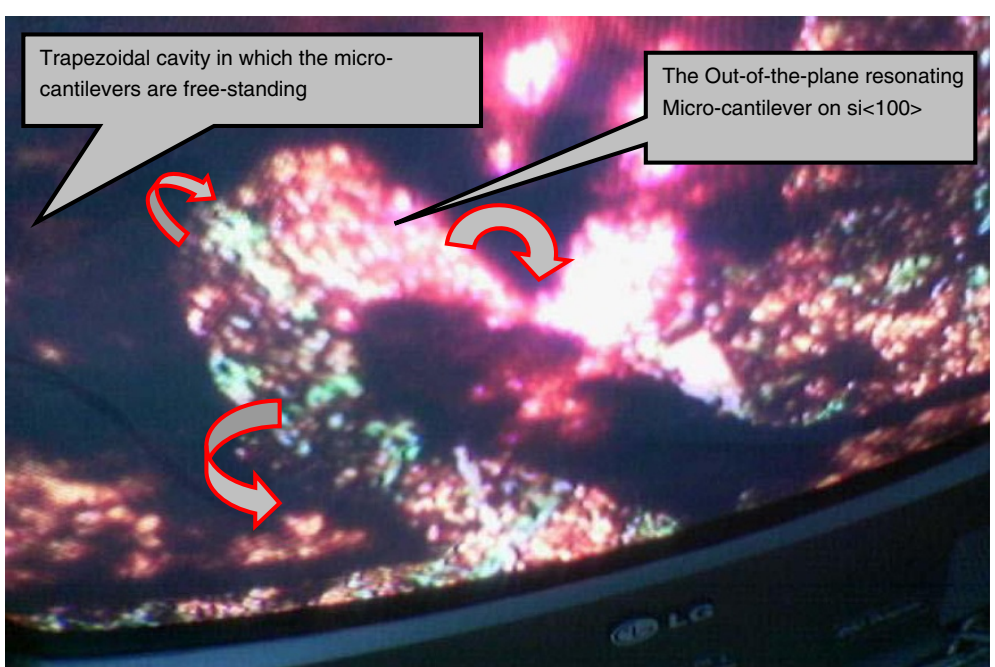

Figure 3 Atomic force microscopy micrograph of surface-functionalized micro-cantilever on silicon $<100>$ surface. The narrow pointer indicates the out-of-the-plane micro-machined micro-cantilever. The wide pointer indicates the trapezoidal micro-cavity, in which the microcantilevers are freestanding. The curved arrows indicate the displacements (bending directions) of the micro-cantilever due to residual stress.

etched. Thus, the change in film step height, using this proposed method, is measured with the relation (1):

$$
\Delta d=\lambda / 2 \cdot \eta
$$

where $\lambda$ is the wavelength of the laser light and $\eta$ is refractive index of the etched layer $(\mathrm{Au}$, refractive index $=0.181$ ). We have measured length, width, and step height using the proposed method, which is relatively easy and accurate (Table 1). The $X, Y$, and $Z$ coordinates of any part of the structure are measured accurately.

\section{Results and discussion}

Figure 1 shows the proposed high-resolution and accurate two-point optical method for image measurement of the micro-cantilever array. Figure 2 shows the SEM micrograph of the freestanding micro-cantilever array in a trapezoidal micro-cavity with a thickness of $200 \mathrm{~nm}$. The micro-cantilever has a wider parameter distribution, and the mean parameter deviation is approximately 10 $\mathrm{nm}$. Further analysis of the SEM image shows that there is some etch product reminiscent at the bottom of the trapezoidal etch pit. However, the sidewalls of the etch pit

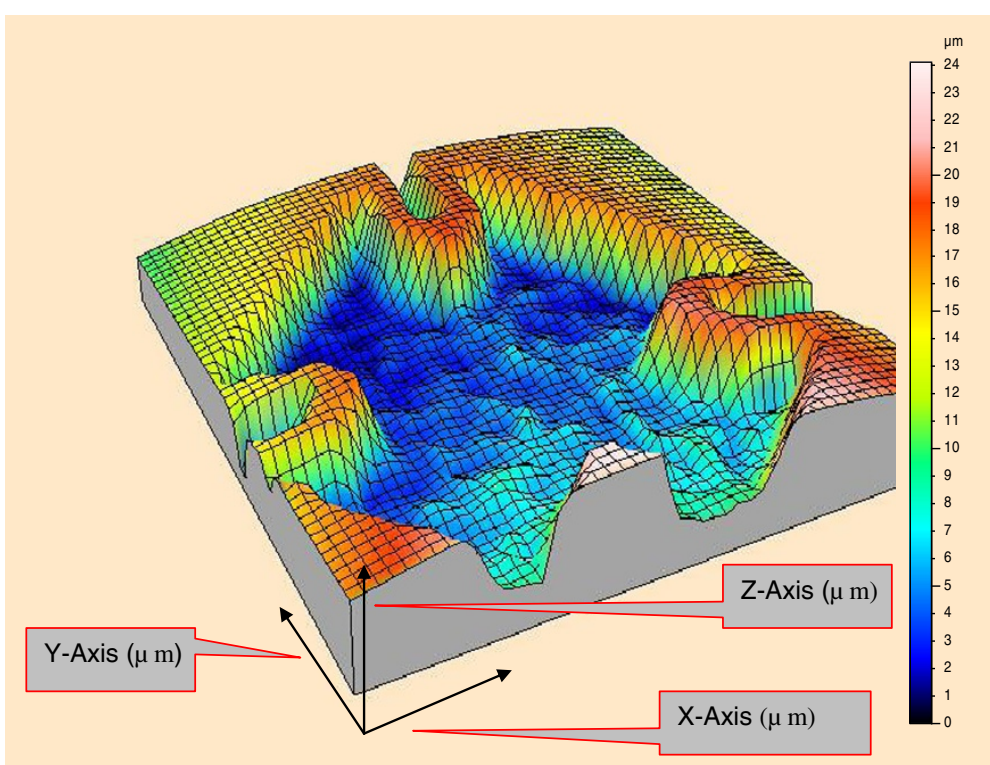

Figure 4 3-D surface profiles (silhouettes) of the micro-cantilever. The $X, Y$, and $Z$ axes are shown by arrows. The origin is placed relatively. 


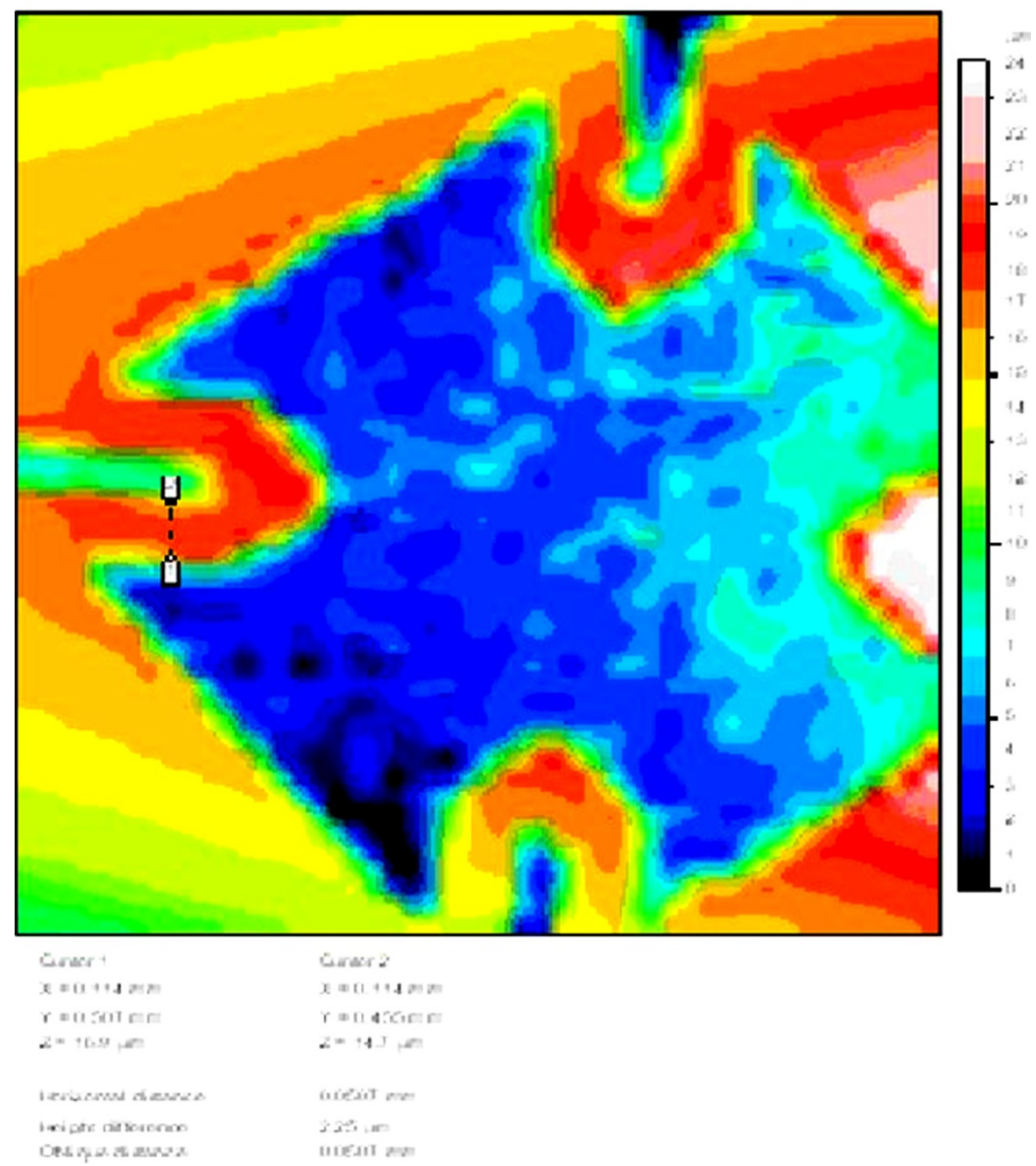

Figure 5 Width measurement of the micro-cantilever. After placing the origin relatively and fixing $Z_{\text {max, }}$ we placed the laser probe at any two points (probe point number indicated in square) for device/feature measurements. The original image obtained has low resolution as is obvious and cannot be further improved.

are smooth. Figure 3 shows the atomic force microscopy image of the sample. It shows that the film with microcantilever arrays is freestanding in a trapezoidal microcavity. A close observation of the AFM image shows clusters of green Y-shaped tentacles of the thiophenol molecule adsorbed on the Au surface. Further analysis shows that the crystallite size distributes in the range of 5 to $10 \mathrm{~nm}$, calculated with the Scherrer formula.

To get the etch depth information, the micro-machined sample was kept on the base of a Taylor Hobson's Form Talysurf 3-D surface profiler machine. The coni-spherical stylus was reset to the original position after choosing the frame of $X-Y$ travel. The machine starts building the etch profile slowly as the coni-spherical stylus moves over the sample. A three-dimensional profile, generated using Taylor Hobson's Form Talysurf ${ }^{\circledast}$ profiler machine, of the sample is shown in Figure 4, using contact mode with the coni-spherical stylus moving parallel to the film plane. The image generated by stylus-based profilometry is in conformity with that by scanning electron microscopy. Figures 5 and 6 depict the width and length measurement with the SEEBREZ ${ }^{\odot}$ machine with high accuracy and repeatable precision, respectively.

A two-dimensional profile to recover the depth information is depicted in Figure 7. The etch profile is very important in assessing the etch uniformity, sidewall smoothness, and etch depth. The etch profile also infers the shape, the slope, and the etch depth of the microcavity, in which the micro-cantilevers are freestanding. It is obvious, from the inspection of etch profile of the trapezoidal micro-cavity, that the sidewalls of this anisotropically etched trapezoidal micro-cavity are smooth 


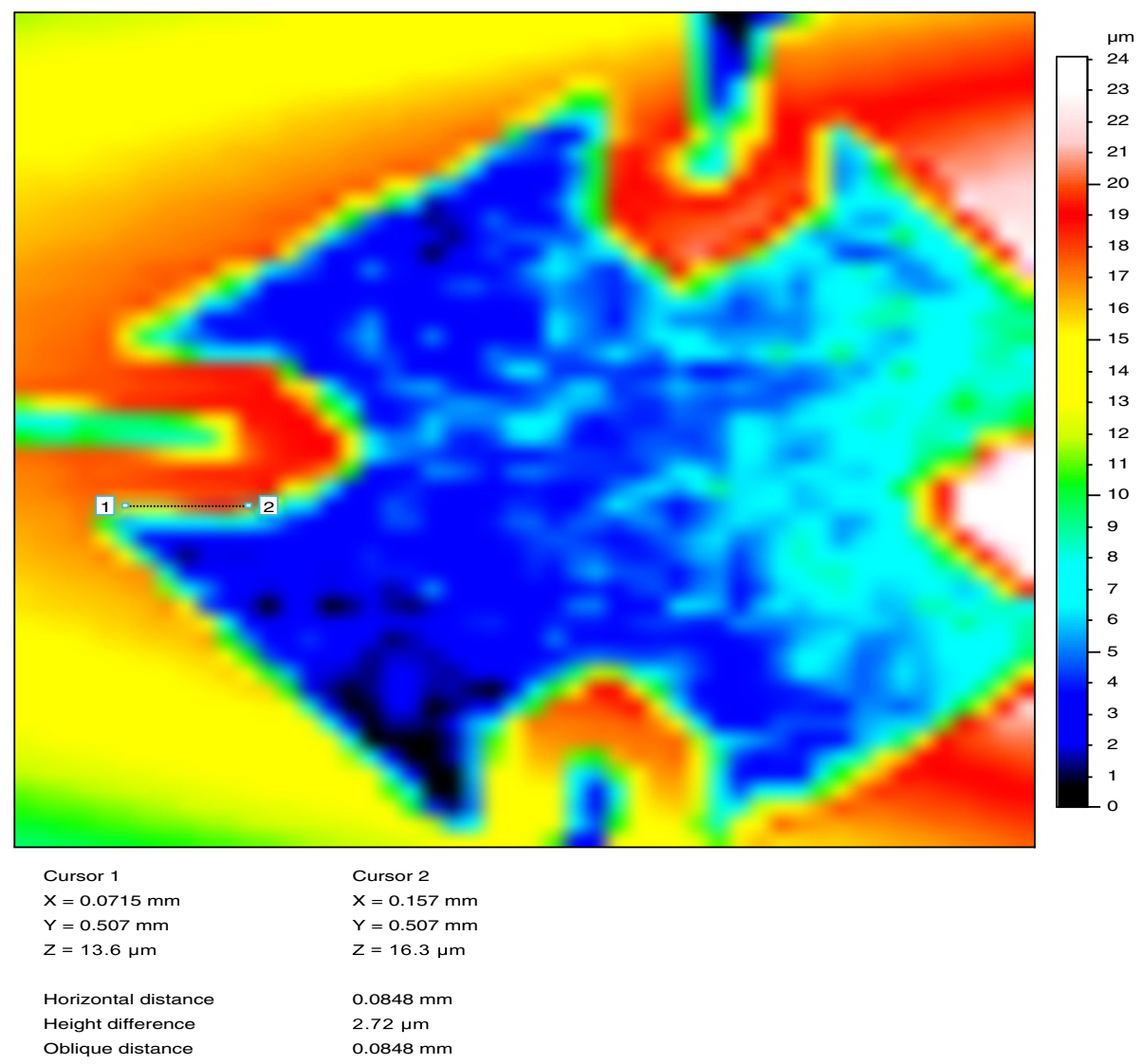

Figure 6 Length measurement of the micro-cantilever. After placing the origin relatively and fixing $Z_{\max }$ we placed the laser probe at any two points (probe point numbers indicated in square) for device/feature measurements. The original image obtained has low resolution as is obvious and cannot be further improved.

since the profile is not jagged. However, the bottom of the anisotropically etched micro-cavity is not smooth since the profile line is jagged containing hills and dales. Inspection of the etch profile and scanning electron micrograph confirms that the sidewalls are smooth and there is some etch product reminiscent at the bottom of the trapezoidal micro-cavity.

\section{Conclusions}

In summary, micro-cantilever arrays have successfully been fabricated on a silicon $<100>$ substrate using bulk micro-machining technique, deposited with a chrome-gold layer using the RF magnetron sputtering method. These micro-cantilever arrays were surface-functionalized using $1 \mu \mathrm{mol}$ thiophenol in ethanol solution for $3 \mathrm{~h}$ and then

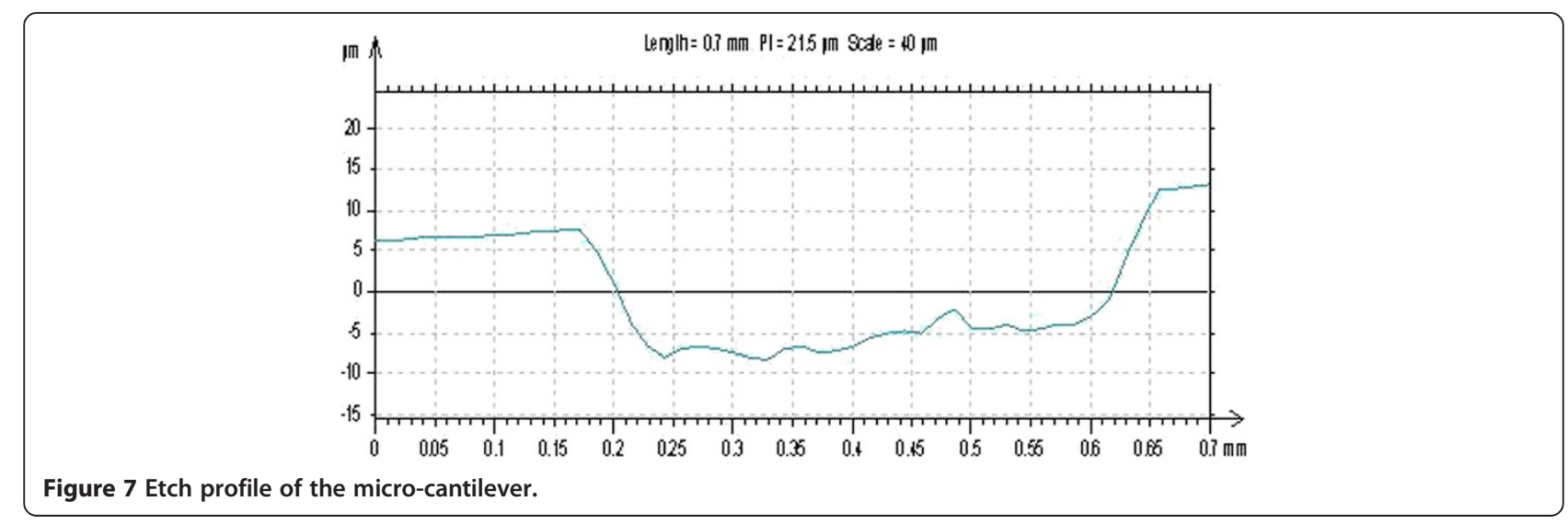


rinsed with ethanol solution for $2 \mathrm{~min}$. The microcantilever surface becomes functionalized for mass sensing. The length, width, and step height measurement of the micro-cantilever is obtained with a non-contact method. We propose a high-resolution and accurate two-point optical method for non-contact measurement of step height, which is a novel method.

The etch profile is very important in assessing the etch uniformity, sidewall smoothness, and etch depth. The etch profile also infers the shape, the slope, and the etch depth of the micro-cavity, in which the micro-cantilevers are freestanding. It is obvious, from the inspection of the etch profile of the trapezoidal micro-cavity, that the sidewalls of this anisotropically etched trapezoidal microcavity are smooth since the profile is not jagged. However, the bottom of the anisotropically etched micro-cavity is not smooth since the profile line is jagged containing hills and dales, where surface tension in the inks or paints causes either rounding or dimpling of the 'step', and the stresses caused by the curing process can cause distortion in the substrate. The curvature of the substrate might well be sufficient to prevent the use of the simple least squares line fit. The step and substrate areas are then treated as line segments, allowing the curvature of the substrate to be removed, resulting in a straight-line representation of the substrate. The step heights are calculated from this line in the areas adjacent to each step.

Inspection of the etch profile and scanning electron micrograph confirms that the sidewalls are smooth and there is some etch product reminiscent at the bottom of the trapezoidal micro-cavity. With this substantial conclusion, we propose a high-resolution and accurate method for the exact measurement of length, width, and etch depth of the micro-machined micro-cantilever. Further, this method can be extended for the measurement of significant parameters of other out-of-the-plane MEMS structures.

The principal limitation with the use of the proposed method is that the minimum probe point separation must be $158.25 \mathrm{~nm}$ and hence shall limit the measurement of features less than $158.25 \mathrm{~nm}$. The minimum number of points would reduce the signal-to-noise ratio that may be offset by the ability to average several scans. The rapid scans would also reduce the sensitivity of the instrument to certain environmental effects, such as mechanical drift due to temperature and vibration.

\section{Competing interests}

The authors did not provide this information.

\section{Authors' contributions}

The authors did not provide this information.

\section{Authors' information}

ASK was born in India in 1966. He received his bachelor's degree from the Amaravati University of India in 1988 and Master in Engineering degree from the Dr. B. A. M. University of India in 1993, both in electronics engineering. He is currently pursuing a Ph.D. degree with the Department of Electrical
Engineering, Indian Institute of Technology, Bombay, India. His research interests include MEMS layout, design, simulation, and fabrication. PRA was born in India in 1947. He received his bachelor's degree from the Indore University of India in 1968 and Master of Technology degree from the Indian Institute of Technology, Kanpur, India, in 1993, both in electronics engineering. He was conferred Ph.D. by the University of Mumbai in 1988 He is currently a professor in the Department of Electrical Engineering, Indian Institute of Technology, Bombay, India. He was a member of the team that made the first TTL IC in India in 1972. Since then, he has more than 20 years experience in MOS/bipolar IC design and fabrication. He has worked at Stanford IC labs for 2 years (1977 to 1978) as a visiting research associate. To his credit, there are 79 publications on silicon technology, which includes journal and international conference papers. His current research interest includes MEMS design, layout, mask-making, fabrication, packaging, testing, reliability, etc.

\section{Acknowledgments}

The authors acknowledge the Microelectronics Group, Nanoelectronics Centre, Department of Electrical Engineering; Department of Chemistry; Department of Physics; and Suman Mashruwala Micromachining Laboratory Department of Mechanical Engineering, Indian Institute of Technology Bombay, Powai, Mumbai, and the Precision Engineering Division, Bhabha Atomic Research Centre, Trombay, Mumbai, India.

Received: 8 October 2012 Accepted: 8 October 2012

Published: 14 May 2013

\section{References}

1. Apte, PR, Vaishnav, UD, Lokhare, SG, Palkar, VR, Pattalwar, SM: Micromechanical components with novel properties. Proc. of SPIE 3321, 287-297 (1996)

2. Madou, MJ: Fundamentals of Micro-fabrication: The Science of Miniaturization. CRC, Boca Raton (2002)

3. Wang, X: Femtosecond laser direct fabrication of metallic cantilevers for micro-corrosion-fatigue test. J. Micromech. Microeng. 17, 1307 (2007)

4. Sun, Z, Weckenmann, A: Reflective properties of typical microstructures under white light interferometer. Meas. Sci. Technol. 22(08), 103 (2011)

5. Deal, B: The oxidation of silicon in dry oxygen, wet oxygen and steam. J. Electrochem. Soc. 10(6), 527-533 (1963)

6. Steinsland, E, Finstad, T, Hanneborg, A: Etch rates of (100), (111) and (110) single-crystal silicon in TMAH measured in situ by laser reflectance interferometer. Sensors and Actuators A: Physical 86(1-2), 73-80 (2000)

7. Seelenbinder, JA, Brown, CW, Urish, DW: Self-assembled monolayers of thiophenol on gold as a novel substrate for surface-enhanced infrared absorption. Appl. Spectrosc. 54(3), 366-370 (2000)

8. Remacle, F, Kryachko, ES: Thiophenol and thiophenol radical and their complexes with gold clusters Au5 and Au6. J. Mol. Struct. 708(1-3), 165-173 (2004)

doi:10.1186/2228-5326-3-33

Cite this article as: Kurhekar and Apte: Step height measurement of surface-functionalized micro-machined micro-cantilever by scanning white light interferometry. International Nano Letters 2013 3:33.

\section{Submit your manuscript to a SpringerOpen ${ }^{\circ}$ journal and benefit from:}

- Convenient online submission

- Rigorous peer review

- Immediate publication on acceptance

- Open access: articles freely available online

- High visibility within the field

- Retaining the copyright to your article

Submit your next manuscript at $>$ springeropen.com 